\title{
Débit plasmatique rénal et filtration glomérulaire chez le mouton vigile : influence d'un régime carencé en protéines
}

\author{
A Cirio, R Boivin \\ Laboratoire de Physiologie, Ecole Nationale Vétérinaire de Lyon, \\ BP 83, 69280 Marcy l'Etoile, France
}

\begin{abstract}
Summary - In conscious sheep fed a low protein diet, the urinary flow rate and glomerular filtration rate were reduced. This reduction was not linked to a low blood urea level.
\end{abstract}

Introduction - Chez le mouton soumis à un régime hypoprotéqque, les données relatives aux variations du débit plasmatique rénal $(D P R)$ et du débit de filtration glomérulaire (DFG) sont contradictoires. Nous avons trouvé une réduction (non significative) de ces 2 paramètres chez le mouton anesthésié (Cirio et Boivin, 1988). Le but de ce travail est de préciser ce phénomène chez le mouton vigile.

Matériel et Méthodes - Sur 14 brebis adultes $(35 \mathrm{à} 50 \mathrm{~kg})$, nous avons utilisé 2 régimes isoénergétiques : standard $(P+: n=8)$ et carencé en protéines ( $\mathrm{P}-: n=6)$, qui apportaient respectivement 48 et $28 \mathrm{~g}$ protéines digestibles intestinales (azote) (PDIN) pour $50 \mathrm{~kg}$ $\mathrm{PV} / \mathrm{f}$. L'aliment a été distribué une fois par j à $9 \mathrm{~h}$ et l'abreuvement s'est fait ad libitum. Le DPR (clairance du p-aminohippurate), le DFG (clairance de l'inuline), l'urémie $\left(U_{p}\right)$ et la clairance $\left(C_{\nu}\right)$ et le taux d'élimination $\left(T E_{\nu}\right)$ rénaux de l'urée, ont été déterminés chez les 2 lots, suivant un protocole classique (Cirio et Boivin, 1988), après 3 semaines de régime expérimental, en 2 j consécutifs ( 6 séances de 15 min/j, 3 le matin, après le repas, et 3 l'après-midi).

Résultats et Discussion - Tous les paramètres mesurés étaient inférieurs (de 42 à $77 \%$ ) chez le groupe $\mathrm{P}$ - par rapport au $P+($ fig 1). Les moutons carencés réduisent donc leur pertes rénales d'urée, en accord avec les données classiques, tout en diminuant leurs $D P R$ et $D F G$. Ce dernier fait, allié à une urémie basse, réduit la charge filtrée d'urée et donc les quantités susceptibles d'être éliminées dans l'urine, ce qui peut partiellement expliquer la chute de la $C_{u}$ et du $T E_{u}$. D'autres auteurs rapportent des résultats dans le même sens chez le mouton vigile (Gans et Mercer, 1962; Rabinowitz et al, 1973), mais la non modification de ces 2 paramètres, dans les mêmes conditions expérimentales, a été signalée par SchmidtNielsen et al (1958) et Maloiy et Scott (1969).

Les DPR et DFG mesurés l'après-midi se sont avérés supérieurs à ceux du matin malgré une urémie plus basse (fig 2). On ne peut donc pas établir de relation directe entre la modification de ces débits et les variations de l'urémie, ce qui est également suggéré par Ergene et Pickering (1978).

En conclusion, les moutons vigiles carencés en protéines réduisent les pertes 

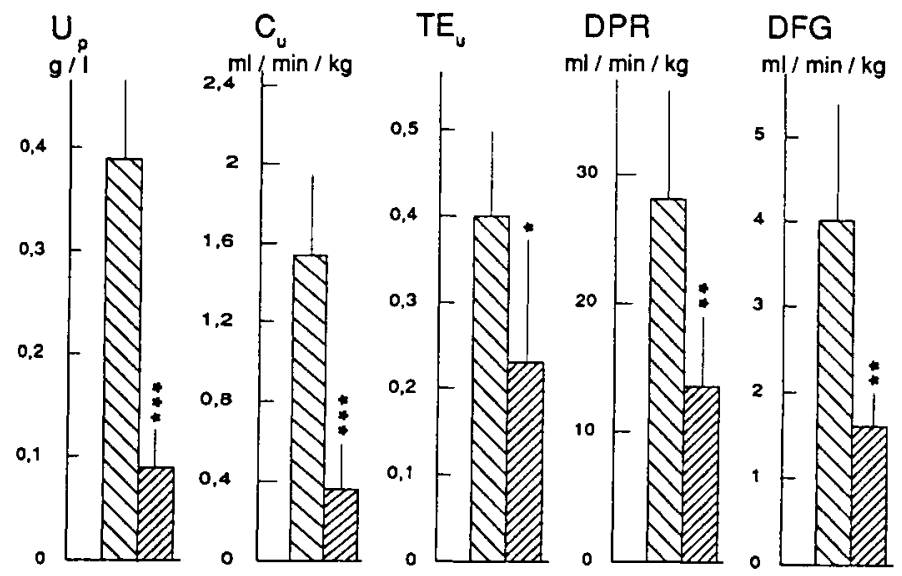

\section{$\nabla \mathrm{P}+$}

ET P -

Fig 1. Urémie $\left(U_{p}\right)$, clairance $\left(C_{u}\right)$ et taux d'élimination $\left(T E_{u}\right)$ rénaux de l'urée, débit plasmatique rénal (DPR) et débit de filtration glomérulaire (DFG), chez les moutons normoprotéiques $(P+: n=8)$ et hypoprotéiques $(P-: n=6)\left(x \pm s ; ;^{*}=P<0,05 ;{ }^{* *}=P<0,01 ;{ }^{* * *}=P<0,001\right)$.
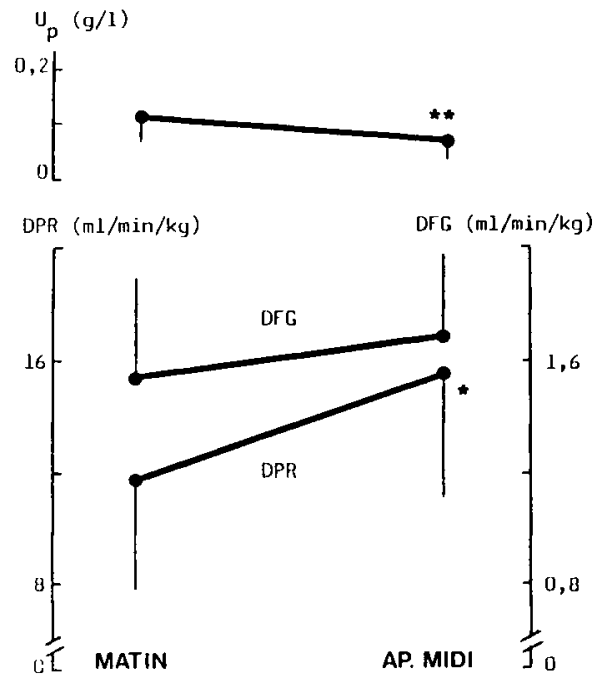

Fig 2. Influence de la prise de nourriture $(9 \mathrm{~h})$ sur l'urémie $\left(U_{p}\right)$, le débit plasmatique rénal (DPR) et le débil de filtration glomérulaire (DFG) mesurés le matin, après le repas, et l'après-midi, chez les moutons hypoprotéiques $(x \pm s ; n=6$; $\left.{ }^{*}=P<0,05 ;{ }^{* *}=P<0,01\right)$. rénales d'urée, en partie par la diminution du volume de plasma traité par le néphron, sans qu'on puisse rattacher ce phénomène à la baisse de l'urémie.

Remerciements - Travail réalisé avec une subvention de l'INRA. Nous remercions le Dr D Grancher pour son aide dans l'élaboration des rations.

Cirio A, Boivin R (1988) J Physiol (Paris) 83, 6473

Ergene N, Pickering EC (1978) Q J Exp Physiol $63,77-81$

Gans JH, Mercer PF (1962) Am J Vet Res 23, 230-234

Maloiy GMO, Scott D (1969) J Physiol (London) 205, 91-101

Rabinowitz L, Gunther RA, Shoji ES, Freedland RA, Avery EH (1973) Kidney Int 4, 188-207

Schmidt-Nielsen B, Osaki $H$, Murdaugh HV Jr, O'Dell R (1958) Am J Physiol 194, 221-228 